\title{
(-)-Epigallocatechin gallate but not chlorogenic acid upregulates osteoprotegerin synthesis through regulation of bone morphogenetic protein- 4 in osteoblasts
}

\author{
KAZUHIKO FUJTA $^{1,2}$, TAKANOBU OTSUKA ${ }^{1}$, NAOHIRO YAMAMOTO ${ }^{1,2}$, \\ SHINGO KAINUMA ${ }^{1,2}$, REOU OHGUCHI ${ }^{1,2}$, TETSU KAWABATA ${ }^{1,2}$, GO SAKAI $^{1,2}$, GEN KUROYANAGI $^{1,2}$, \\ RIE MATSUSHIMA-NISHIWAKI ${ }^{2}$, OSAMU KOZAWA ${ }^{2}$ and HARUHIKO TOKUDA ${ }^{2,3}$ \\ ${ }^{1}$ Department of Orthopedic Surgery, Nagoya City University Graduate School of Medical Sciences, Nagoya, \\ Aichi 467-8601; ${ }^{2}$ Department of Pharmacology, Gifu University Graduate School of Medicine, Gifu 501-1194; \\ ${ }^{3}$ Department of Clinical Laboratory, National Center for Geriatrics and Gerontology, Obu, Aichi 474-8511, Japan
}

Received April 25, 2016; Accepted March 3, 2017

DOI: $10.3892 / \mathrm{etm} .2017 .4491$

\begin{abstract}
Chlorogenic acid (CGA) is a primary phenolic component of coffee and (-)-epigallocatechin gallate (EGCG) is a primary flavonoid component of green tea, both of which have been documented to possess beneficial health properties. A previous study by the present authors demonstrated that $\mathrm{p} 38$ mitogen-activated protein kinase (MAPK) may be associated with osteoprotegerin synthesis stimulated by bone morphogenetic protein-4 (BMP-4) in osteoblast-like MC3T3-E1 cells. In the present study, the effects of CGA and EGCG on BMP-4-stimulated osteoprotegerin synthesis in MC3T3-E1 cells were investigated. It was observed that CGA had no effect on osteoprotegerin release stimulated by BMP-4, whereas EGCG significantly enhanced BMP-4-stimulated osteoprotegerin release $(\mathrm{P}=0.003)$. Levels of osteoprotegerin mRNA expression induced by BMP-4 were also significantly increased by EGCG $(\mathrm{P}=0.03)$. By contrast, EGCG had no significant effect on phosphorylation of Smad1 or p38 MAPK induced by BMP-4. In addition, EGCG had little effect on BMP-induced phosphorylation of p70 S6 kinase; however rapamycin, as an inhibitor of p70 S6 kinase, significantly suppressed osteoprotegerin release $(\mathrm{P}=0.007)$. These data suggest that EGCG but not CGA may upregulate the synthesis of osteoprotegerin induced by BMP-4 in osteoblasts.
\end{abstract}

Correspondence to: Dr Haruhiko Tokuda, Department of Clinical Laboratory, National Center for Geriatrics and Gerontology, 7-430 Morioka-cho, Obu, Aichi 474-8511, Japan

E-mail: tokuda@ncgg.go.jp

Key words: (-)-epigallocatechin gallate, chlorogenic acid, bone morphogenic protein-4, osteoprotegerin, osteoblast

\section{Introduction}

Osteoprotegerin is secreted from osteoblasts and has an inhibitory effect on the differentiation and activation of osteoclasts (1). Bone metabolism is principally regulated by osteoblasts and osteoclasts, which are responsible for the formation and resorption of bone, respectively (2). Through the bone remodeling activities of osteoblasts and osteoclasts, bone tissue in the skeleton is continuously regenerated and renewed (3). Bone remodeling begins with the resorption of bone by osteoclasts, followed by osteoblastic bone formation (4). It has been established that a decrease in bone mineral density results from an imbalance in the bone remodeling process (3).

Dysfunction in bone remodeling leads to metabolic bone disease associated with an increased risk of fracture, including osteoporosis (5). Paget's disease of bone is a common disorder characterized by focal areas of increased and disorganized bone remodeling (6).

Osteoprotegerin and its cognate ligand receptor activator of nuclear factor- $\mathrm{KB}$ (RANK) belong to the tumor necrosis factor receptor family. Osteoprotegerin binds to RANK ligand as a decoy receptor and prevents RANK ligand from binding to RANK, resulting in the suppression of bone resorption through the inhibition of osteoclast differentiation (1). Through this mechanism, the RANK/RANK ligand/osteoprotegerin axis is considered to be an essential regulatory system in the formation of osteoclasts (7).

Chlorogenic acid (CGA), which is a phenolic compound and a main component of coffee, and (-)-epigallocatechin gallate (EGCG), which is a polyphenolic compound and a primary constituent of green tea, are considered to have beneficial properties for human health, including anti-oxidative, anti-inflammatory and anti-cancer properties (8-10). CGA has been documented to increase mineralization in rat tibia and improve mechanical properties of the femoral diaphysis (11). In addition, CGA may suppress osteoclastic bone resorption by downregulating the effects of RANK ligand (12). Similarly, EGCG may inhibit osteoclastic bone resorption and supports 
osteoblastic bone formation $(13,14)$, and green tea consumption may be associated with reduced age-related bone loss and fractures in the elderly (13). However, the underlying molecular mechanisms regarding the effects of CGA and EGCG on bone metabolism are currently unknown.

Bone morphogenetic proteins (BMPs), which belong to the transforming growth factor (TGF)- $\beta$ superfamily, including TGF- $\beta$ and activin, promote bone formation by stimulating the proliferation and differentiation of osteoblasts $(15,16)$. Intracellular signaling activities of BMPs generally occur through a Smad (Smad1/5/8)-dependent pathway (15), although previous evidence suggests that BMP signaling may also occur through a Smad-independent pathway involving the mitogen-activated protein kinase (MAPK) family $(17,18)$. A previous study by the present authors demonstrated that BMP-4 may stimulate the synthesis of osteoprotegerin in osteoblast-like MC3T3-E1 cells, and that p38 MAPK may act as a positive regulator in osteoprotegerin synthesis (19). In the present study, the effects of CGA and EGCG on osteoprotegerin synthesis mediated by BMP-4 in osteoblast-like MC3T3-E1 cells were investigated. It was observed that EGCG but not CGA enhanced osteoprotegerin synthesis stimulated by BMP-4 in osteoblasts.

\section{Materials and methods}

Materials. CGA and EGCG were purchased from Sigma-Aldrich (Merck KGaA, Darmstadt, Germany). BMP-4 and a mouse osteoprotegerin enzyme-linked immunosorbent assay (ELISA) kit (cat. no. MOP00) were obtained from R\&D Systems, Inc. (Minneapolis, MN, USA). Rapamycin was obtained from Merck KGaA. Antibodies against phosphorylated Smad1 (cat. no. 13820), p38 MAPK (cat. no. 9212), phosphorylated (phospho)-p38 MAPK (cat. no. 4511), p70 S6 kinase (cat. no. 9202) and phospho-p70 S6 kinase (cat. no. 9205S) were obtained from Cell Signaling Technology, Inc. (Danvers, MA, USA). Antibodies against GAPDH (cat. no. sc-25778) were purchased from Santa Cruz Biotechnology, Inc. (Dallas, TX, USA). Secondary antibodies, peroxidase-labeled goat anti-rabbit immunoglobulin G (cat. no. 074-1506) were purchased from Seracare (Milford, MA, USA). An enhanced chemiluminescence (ECL) western blotting detection system was obtained from GE Healthcare Life Sciences (Chalfont, UK). EGCG and rapamycin were individually diluted in dimethyl sulfoxide $(100 \mathrm{mM})$ and CGA was diluted in ethanol (100 mM). Both DMSO and ethanol were used at a maximum concentration of $0.1 \%$, which did not affect the assay for osteoprotegerin or western blot analysis.

Cell culture. Cloned osteoblast-like MC3T3-E1 cells derived from newborn mouse calvaria (20) were maintained as described previously (21). Briefly, cells were seeded into $35 \mathrm{~mm}$ diameter dishes $\left(5 \times 10^{4}\right.$ cells/dish) for ELISA and reverse transcription-quantitative polymerase chain reaction (RT-qPCR) experiments or $90 \mathrm{~mm}$ diameter dishes $\left(2 \times 10^{5}\right.$ cells/dish) for Western blotting and cultured in $\alpha$-minimum essential medium ( $\alpha$-MEM; cat. no. M8042, Sigma-Aldrich; Merck KGaA) containing $10 \%$ fetal bovine serum (FBS; cat. no. 12483-020; Gibco; Thermo Fisher Scientific Inc. Waltham, MA, USA) at $37^{\circ} \mathrm{C}$ in a humidified atmosphere of $5 \% \mathrm{CO}_{2} / 95 \%$ air. After
5 days, the medium was replenished with $\alpha$-MEM containing $0.3 \% \mathrm{FBS}$ at $37^{\circ} \mathrm{C}$ for $48 \mathrm{~h}$. Cells were then used in the following experiments.

Assay for osteoprotegerin. Cultured MC3T3-E1 cells were pretreated with $1,3,10,30$ or $50 \mu \mathrm{M}$ of CGA or 1,3 or $10 \mu \mathrm{M}$ of EGCG at $37^{\circ} \mathrm{C}$ for $60 \mathrm{~min}$, then stimulated with $30 \mathrm{ng} / \mathrm{ml}$ BMP-4 or PBS supplemented with $0.01 \%$ bovine serum albumin (Sigma-Aldrich; Merck KGaA) containing $0.1 \%$ ethanol (as a vehicle) in $1 \mathrm{ml} \alpha$-MEM containing $0.3 \% \mathrm{FBS}$ at $37^{\circ} \mathrm{C}$ for $0,12,24,36$ or $48 \mathrm{~h}$. The conditioned medium was collected following the incubation periods and the concentration of osteoprotegerin was measured using the mouse osteoprotegerin ELISA kit, according to the manufacturer's protocol.

RT-qPCR. Cultured MC3T3-E1 cells were pretreated with $10 \mu \mathrm{M}$ EGCG or vehicle at $37^{\circ} \mathrm{C}$ for $60 \mathrm{~min}$, then stimulated with $70 \mathrm{ng} / \mathrm{ml} \mathrm{BMP}-4$ or vehicle in $1 \mathrm{ml}$ of $\alpha$-MEM containing $0.3 \% \mathrm{FBS}$ at $37^{\circ} \mathrm{C}$ for $6 \mathrm{~h}$. Total RNA was isolated by TRIzol reagent (Invitrogen; Thermo Fisher Scientific, Inc., Waltham, MA, USA) and was reverse transcribed into complementary DNA using an Omniscript Reverse Transcriptase kit (Qiagen Inc., Valencia, CA, USA) according to the manufacturer's protocol. qPCR was performed using a LightCycler Capillary system and Fast Start DNA Master SYBR-Green I kit (Roche Diagnostics, Basel, Switzerland). The forward and reverse primers for mouse osteoprotegerin mRNA were purchased from Takara Biotechnology Co., Ltd. (Dalian, China). The sequences were as follows: Forward 5'-CAATGGCTGGCT TGGTTTCATAG-3' and reverse 5'-CTGAACCAGACATGA CAGCTGGA-3'. The forward and reverse primers for mouse GAPDH mRNA were synthesized based on the report of Simpson et al (22) and obtained from Sigma-Aldrich (Merck $\mathrm{KGaA}$ ). The sequences were as follows: Forward 5'-AACGAC CCCTTCATTGAC-3' and reverse 5'-TCCACGACATACTCA GCAC-3'. The reaction mixtures were incubated at $95^{\circ} \mathrm{C}$ for $10 \mathrm{~min}$, followed by 40 cycles at $60^{\circ} \mathrm{C}$ for $5 \mathrm{sec}$ and $72^{\circ} \mathrm{C}$ for $7 \mathrm{sec}$. The amplified products were determined by a melting curve analysis (23) according to the system protocol. The data were analyzed by second derivative maximum method using LyghtCycler3 Data Analysis (Version 3.5.28; Roche Diagnostics). Levels of osteoprotegerin mRNA were normalized to those of GAPDH mRNA.

Western blot analysis. Cultured MC3T3-E1 cells were pretreated with 10,20 or $30 \mu \mathrm{M}$ of EGCG at $37^{\circ} \mathrm{C}$ for $60 \mathrm{~min}$, then stimulated with $30 \mathrm{ng} / \mathrm{ml} \mathrm{BMP}-4$ or vehicle, PBS supplemented with $0.01 \%$ bovine serum albumin containing $0.1 \%$ ethanol, in $1 \mathrm{ml}$ of $\alpha$-MEM containing $0.3 \% \mathrm{FBS}$ at $37^{\circ} \mathrm{C}$ for 45,90 or 120 min according to our previous reports $(19,24-26)$. Cells were then washed twice with phosphate-buffered saline and lysed, homogenized and sonicated with 20 short $1 \mathrm{sec}$ bursts using a TOMY Ultrasonic Disruptor UD-211 (TOMY Digital Biology, Co., Ltd., Tokyo, Japan) in a lysis buffer containing $62.5 \mathrm{mM}$ Tris/ $\mathrm{HCl}(\mathrm{pH} 6.8) 2 \%$ sodium dodecyl sulfate (SDS), $50 \mathrm{mM}$ dithiothreitol and $10 \%$ glycerol. SDS-PAGE was performed according to the Laemmli method (27) on $10 \%$ polyacrylamide gels. The proteins were fractionated and transferred onto Immuno-Blot polyvinyl difluoride (PVDF) 
membranes (Bio-Rad Laboratories, Inc., Hercules, CA, USA). The membranes were blocked with $5 \%$ fat-free dry milk in Tris-buffered saline-Tween-20 [TBS-T; 20 mM Tris- $\mathrm{HCl}(\mathrm{pH}$ 7.6) $137 \mathrm{mM} \mathrm{NaCl}$ and $0.1 \%$ Tween-20) at room temperature for $2 \mathrm{~h}$ prior to incubation with primary antibodies. Western blot analysis was performed as described previously (28) using antibodies against phospho-Smad1, GAPDH, phospho-p70 S6 kinase, p70 S6 kinase, phospho-p38 MAPK and p38 MAPK as primary antibodies and peroxidase-labeled goat anti-rabbit immunoglobulin $\mathrm{G}$ as secondary antibodies. The primary and secondary antibodies were diluted to $1: 1,000$ with 5\% fat-free dry milk in TBS-T. Peroxidase activity on the PVDF membranes was detected on an X-ray film using the ECL western blotting detection system.

Measurements. The absorbance of enzyme immunosorbent assay samples was measured at $450 \mathrm{~nm}$ with an EL 340 Bio Kinetic Reader (BioTek Instruments, Inc., Winooski, VT, USA). Densitometric analysis of western blotting was performed using a scanner and Image J 1.47 software (National Institutes of Health, Bethesda, MD, USA). Levels of phosphorylated protein were calculated as the background-subtracted signal intensity of each phosphorylation signal normalized to the respective total protein signal, and plotted as a fold increase relative to that of control cells treated with vehicle reagent.

Statistical analysis. Data were analyzed using one-way analysis of variance followed by a Bonferroni method for multiple comparisons between pairs, using Microsoft Office Excel 2013 for Windows (Microsoft Corporation, Redmond, WA, USA) and $\mathrm{P}<0.05$ was considered to indicate a statistically significant difference. All data are presented as the mean \pm standard error of the mean of three replicate experiments from three independent cell preparations.

\section{Results}

Differential effects of CGA or EGCG on BMP-4-stimulated osteoprotegerin release in MC3T3-E1 cells. In a previous study by the present authors (19), it was demonstrated that BMP-4 stimulates osteoprotegerin synthesis in osteoblast-like MC3T3-E1 cells. The present study initially investigated the effect of CGA on BMP-4-stimulated osteoprotegerin release in MC3T3-E1 cells. It was observed that CGA $(\leq 50 \mathrm{nM})+\mathrm{BMP}-4$ had no significant effect on BMP-4-stimulated osteoprotegerin release in comparison to the value of vehicle + BMP-4 (Fig. 1).

The effect of EGCG on BMP-4-stimulated osteoprotegerin release was also investigated in MC3T3-E1 cells. In contrast to CGA, it was observed that $30 \mu \mathrm{M}$ of EGCG + BMP-4 (Fig. 2A) significantly enhanced BMP-4-stimulated osteoprotegerin release in a time-dependent manner compared with the value of vehicle + BMP-4 $(\mathrm{P}<0.005$; Fig. 2A). In addition, the stimulatory effects of EGCG + BMP-4 (Fig. 2B) on osteoprotegerin release were dose-dependent between 1 and $10 \mu \mathrm{M}$ in comparison to the value of vehicle $+\mathrm{BMP}-4(\mathrm{P}<0.003$; Fig. 2B).

EGCG stimulates BMP-4-induced osteoprotegerin mRNA expression in MC3T3-E1 cells. To determine whether the

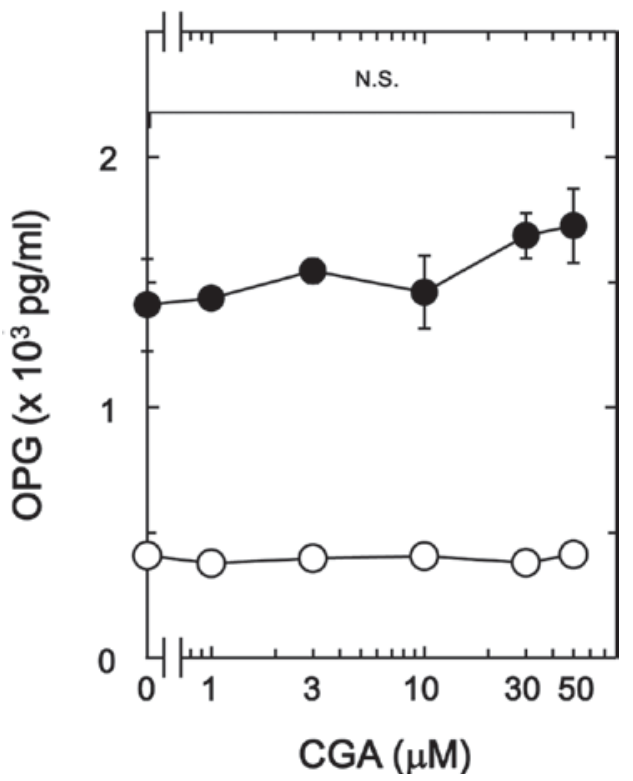

Figure 1. Effect of CGA on BMP-4-stimulated OPG release in MC3T3-E1 cells. The cultured cells were pretreated with the indicated doses of CGA for $60 \mathrm{~min}$, then stimulated with $30 \mathrm{ng} / \mathrm{ml} \mathrm{BMP}-4$ or vehicle $(\bullet)$ or vehicle (o) for $48 \mathrm{~h}$. Levels of OPG in the culture medium were determined by ELISA. Data are presented as the mean \pm standard error of the mean of three replicate experiments from three independent cell preparations. CGA, chlorogenic acid, BMP-4, bone morphogenic protein-4; OPG, osteoprotegrin; N.S., no significant difference.

stimulatory effects of EGCG on BMP-4-stimulated osteoprotegerin release was mediated by transcriptional events in osteoblast-like MC3T3-E1 cells, the effect of EGCG on BMP-4-induced expression of osteoprotegerin mRNA was subsequently evaluated. It was observed that EGCG $(10 \mu \mathrm{M})+\mathrm{BMP}-4$ significantly enhanced BMP-4-induced osteoprotegerin mRNA expression in comparison to the value of vehicle + BMP-4 (P=0.03; Fig. 3).

EGCG has no effect on BMP-4-induced phosphorylation of Smad1. The Smad1/5/8 pathway is an intracellular signal transduction system that principally mediates the effects of BMPs (15). To determine whether the stimulatory effects of EGCG on osteoprotegerin synthesis were dependent on the activation of Smad1/5/8 in MC3T3-E1 cells, the present study evaluated the effect of EGCG on BMP-4-induced phosphorylation of Smad1. It was observed that vehicle alone or EGCG + vehicle did not stimulate the phosphorylation of Smad1, whereas vehicle + BMP-4 did. It was observed that EGCG (10-30 $\mu \mathrm{M})+$ BMP-4 had no effect on BMP-4-induced phosphorylation of Smad1 in comparison to the value of vehicle + BMP-4 (Fig. 4)

EGCG has little effect on BMP-4-induced phosphorylation of $p 38 M A P K$. In previous studies by our group $(19,24)$, BMP-4 induced the activation of p38 MAPK in osteoblast-like MC3T3-E1 cells, and p38 MAPK was associated with the BMP-4-stimulated osteoprotegerin synthesis in these cells. Therefore, to determine whether the effect of EGCG on BMP-4-stimulated osteoprotegerin synthesis was associated with activation of p38 MAPK in MC3T3-E1 cells, the present study evaluated the effect of EGCG on BMP-4-induced 

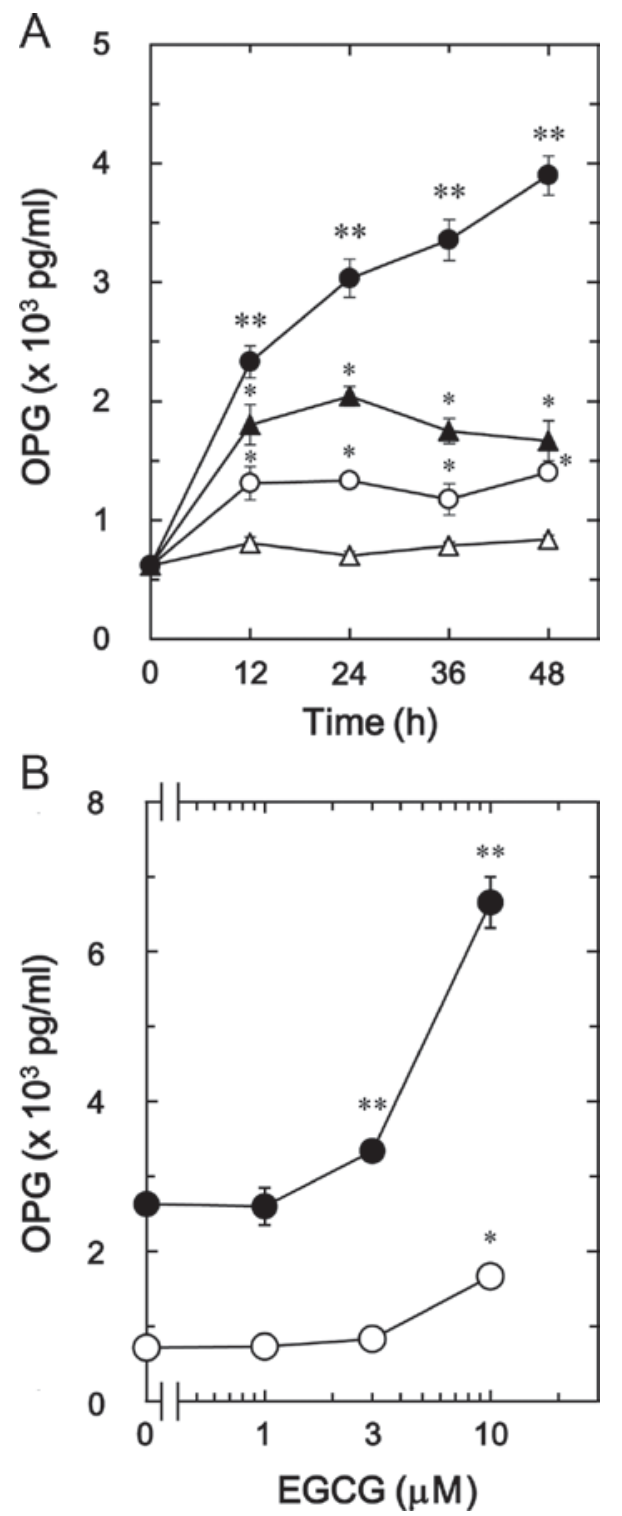

Figure 2. Effect of EGCG on BMP-4-stimulated OPG release in MC3T3-E1 cells. (A) Cultured cells were pretreated with $30 \mu \mathrm{M}$ EGCG $(\bullet, \circ)$ or vehicle $(\boldsymbol{\Lambda}, \Delta)$ for $60 \mathrm{~min}$, then stimulated with $30 \mathrm{ng} / \mathrm{ml} \mathrm{BMP}-4(\bullet, \boldsymbol{\Lambda})$ or vehicle $(\circ, \Delta)$ for the indicated time periods. (B) Cultured cells were pretreated with the indicated doses of EGCG for $60 \mathrm{~min}$, then stimulated with $30 \mathrm{ng} / \mathrm{ml} \mathrm{BMP}-4(\bullet)$ or vehicle ( $($ ) for $48 \mathrm{~h}$. Levels of osteoprotegerin in the culture medium were determined by ELISA. Data are presented as the mean \pm standard error of the mean of three replicate experiments from three independent cell preparations. ${ }^{*} \mathrm{P}<0.05$ vs. control cells and ${ }^{* *} \mathrm{P}<0.05$ vs. cells stimulated by BMP-4 and pretreated with vehicle. EGCG, (-)-epigallocatechin gallate; BMP-4, bone morphogenic protein-4; OPG, osteoprotegrin.

phosphorylation of p38 MAPK. It was observed that EGCG $(10-30 \mu \mathrm{M})+$ BMP-4 had no significant effect on BMP-4-induced phosphorylation of $\mathrm{p} 38$ MAPK in comparison to the value of vehicle + BMP-4 (Fig. 5).

Rapamycin suppresses BMP-4-stimulated osteoprotegerin release in MC3T3-El cells. BMP-4 has been demonstrated to induce the synthesis of vascular endothelial growth factor through p70 S6 kinase in osteoblast-like MC3T3-E1 cells (25). Therefore, the present study also evaluated whether p70 S6 kinase serves a role in BMP-4-stimulated osteoprotegerin synthesis in MC3T3-E1 cells. It was observed that rapamycin,

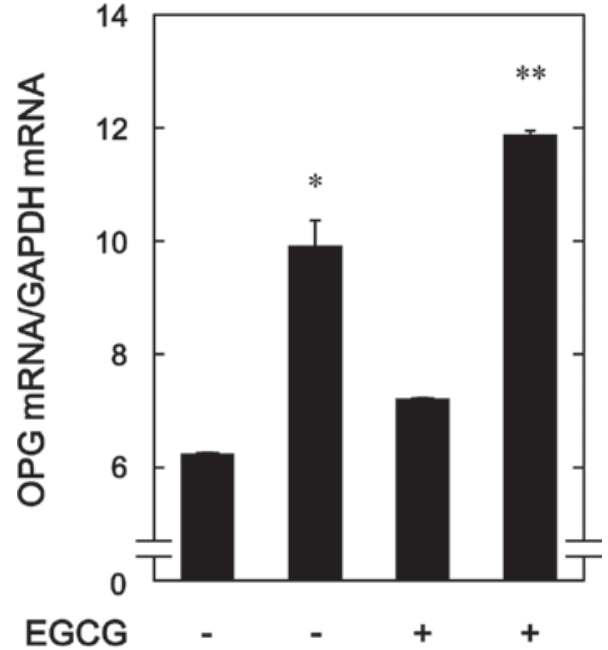

Figure 3. Effect of EGCG on BMP-4-induced expression of OPG mRNA in MC3T3-E1 cells. Cultured cells were pretreated with $10 \mu \mathrm{M}$ EGCG or vehicle for $60 \mathrm{~min}$, then stimulated with $30 \mathrm{ng} / \mathrm{ml} \mathrm{BMP}-4$ or vehicle for $6 \mathrm{~h}$. Total RNA was isolated and quantified using reverse transcription-quantitative polymerase chain reaction. Data are presented as the mean \pm standard error of the mean of three replicate experiments from three independent cell preparations ${ }^{*} \mathrm{P}<0.05$ vs. control cells and ${ }^{* *} \mathrm{P}<0.05$ vs. cells stimulated by BMP-4 and pretreated with vehicle. EGCG, (-)-epigallocatechin gallate; BMP-4, bone morphogenic protein-4; OPG, osteoprotegrin.
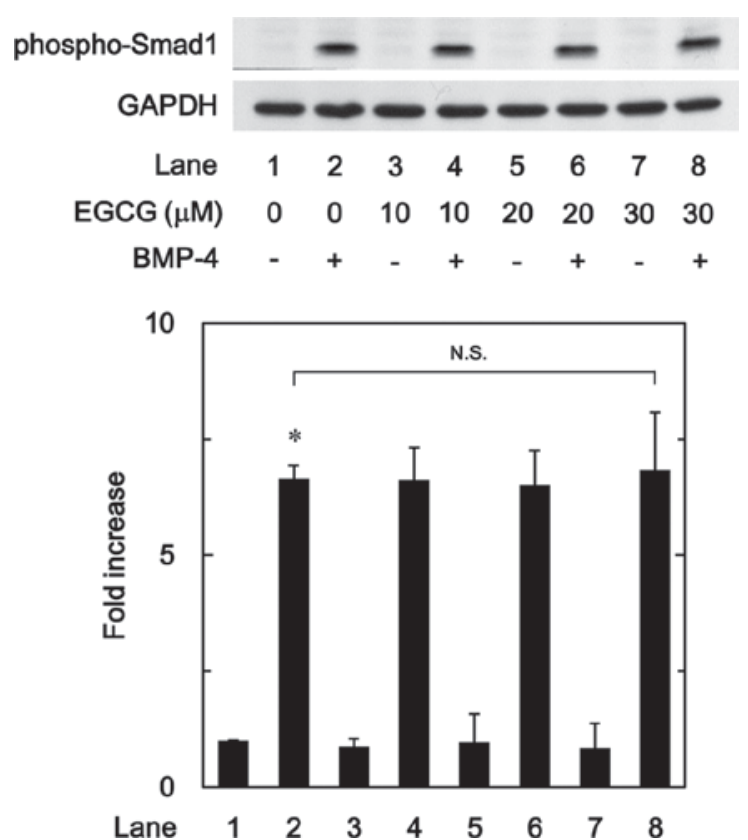

Figure 4. Effect of EGCG on BMP-4-induced phosphorylation of Smad1 in MC3T3-E1 cells. Cultured cells were pretreated with the indicated doses of EGCG for $60 \mathrm{~min}$, then stimulated with $30 \mathrm{ng} / \mathrm{ml} \mathrm{BMP}-4$ or vehicle for $45 \mathrm{~min}$. Cell extracts were then subjected to SDS-PAGE and subsequent western blot analysis using primary antibodies against phospho-Smad1 and GAPDH. The bar chart shows the levels of BMP-4-induced phosphorylation determined by laser-based densitometric analysis. Data are presented as the mean \pm standard error of the mean of three replicate experiments from three independent cell preparations. ${ }^{*} \mathrm{P}<0.05$ vs. control cells. N.S., no significant difference; EGCG, (-)-epigallocatechin gallate; BMP-4, bone morphogenic protein-4; phospho, phosphorylated.

as an inhibitor of mammalian target of rapamycin (mTOR) that activates p70 S6 kinase (29), significantly suppressed BMP-4-stimulated osteoprotegerin release in a dose-dependent 

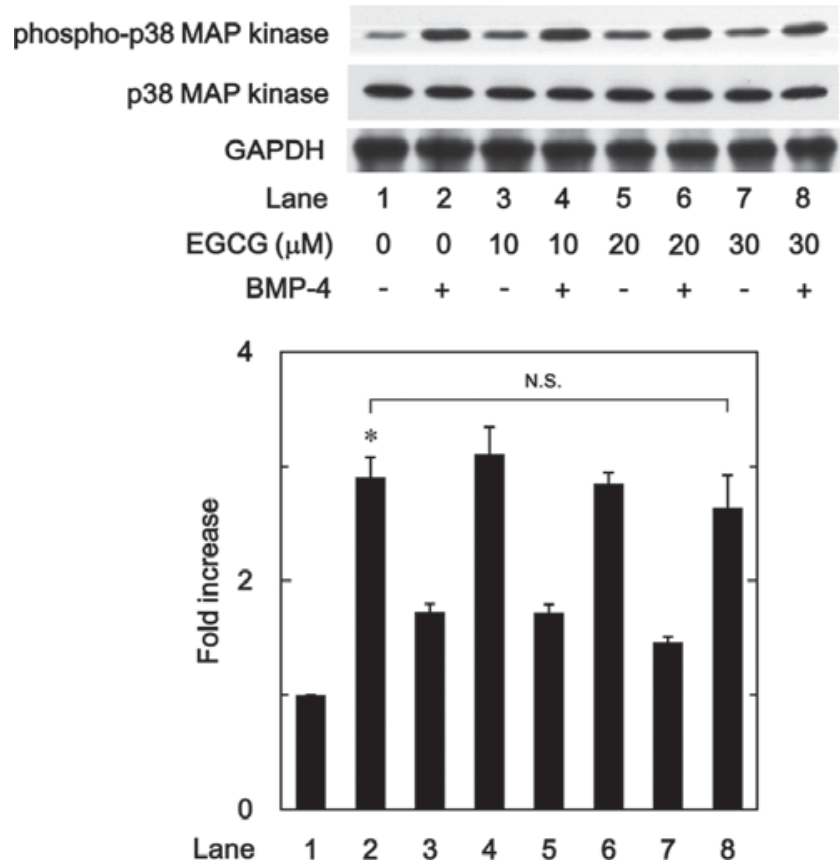

Figure 5. Effect of EGCG on BMP-4-induced phosphorylation of p38 MAPK in MC3T3-E1 cells. Cultured cells were pretreated with various doses of EGCG for $60 \mathrm{~min}$, then stimulated with $30 \mathrm{ng} / \mathrm{ml}$ BMP-4 or vehicle for $90 \mathrm{~min}$. Cell extracts were then subjected to SDS-PAGE and subsequent western blot analysis using antibodies against phospho-p38 MAPK and p38 MAPK. The bar chart shows the levels of BMP-4-induced phosphorylation determined by laser-based densitometric analysis. Data are presented as the mean \pm standard error of the mean of three replicate experiments from three independent cell preparations. " $\mathrm{P}<0.05$ vs. control cells treated with vehicle alone. N.S., no significant difference; EGCG, (-)-epigallocatechin gallate; BMP-4, bone morphogenic protein-4; p38 MAPK, p38 mitogen-activated protein kinase; phospho, phosphorylated.

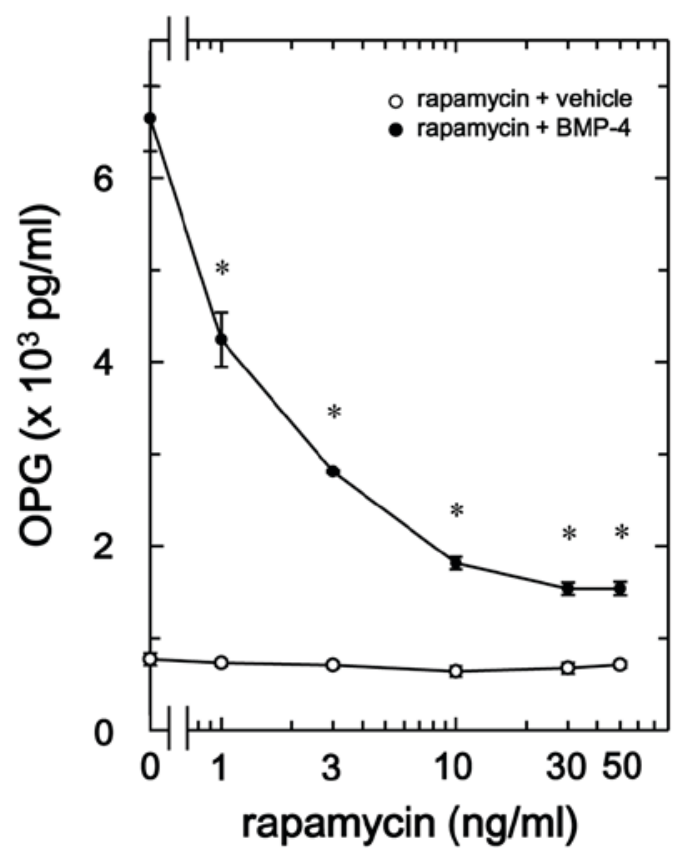

Figure 6. Effect of rapamycin on BMP-4-stimulated OPG release in MC3T3-E1 cells. Cultured cells were pretreated with the indicated doses of rapamycin for $60 \mathrm{~min}$, then stimulated with $30 \mathrm{ng} / \mathrm{ml} \mathrm{BMP}-4(\bullet)$ or vehicle (०) for $48 \mathrm{~h}$. Levels of OPG in the culture medium were determined by ELISA. Data are presented as the mean \pm standard error of the mean of three replicate experiments from three independent cell preparations. ${ }^{~} \mathrm{P}<0.05$ vs. BMP-4 alone. BMP-4, bone morphogenic protein-4; OPG, osteoprotegrin.
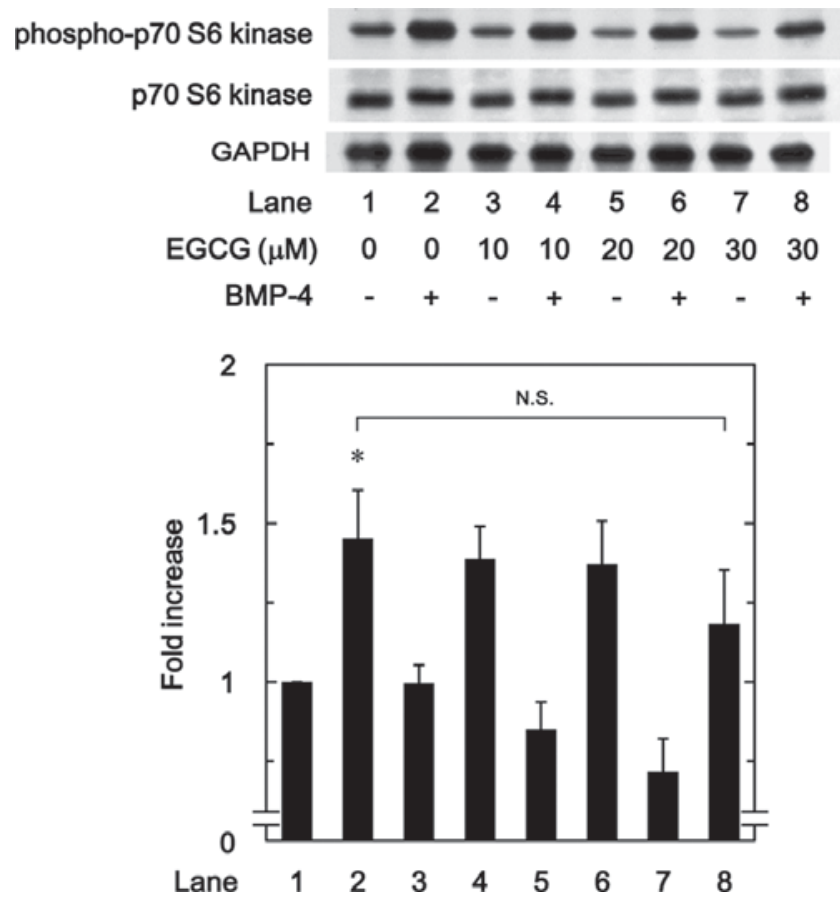

Figure 7. Effect of EGCG on BMP-4-induced phosphorylation of p70 S6 kinase in MC3T3-E1 cells. Cultured cells were pretreated with the indicated doses of EGCG for $60 \mathrm{~min}$, then stimulated with $30 \mathrm{ng} / \mathrm{ml} \mathrm{BMP}-4$ or vehicle for $120 \mathrm{~min}$. Cell extracts were then subjected to SDS-PAGE and subsequent western blot analysis using antibodies against phospho-p70 S6 kinase and p70 S6 kinase. The bar chart shows the levels of BMP-4-induced phosphorylation determined by laser-based densitometric analysis. Data are presented as the mean \pm standard error of the mean of three replicate experiments from three independent cell preparations. ${ }^{*} \mathrm{P}<0.05$ vs. control cells treated with vehicle alone. N.S., no significant difference; EGCG, (-)-epigallocatechin gallate; BMP-4, bone morphogenic protein-4; phospho, phosphorylated.

manner between 1 and $50 \mathrm{ng} / \mathrm{ml}$ in comparison to the value of vehicle + BMP-4 (P<0.007; Fig. 6).

EGCG has little effect on BMP-4-induced phosphorylation of p70 S6 kinase. To determine whether the effect of EGCG on BMP-4-stimulated osteoprotegerin synthesis was due to the activation of p70 S6 kinase in MC3T3-E1 cells, the present study evaluated the effect of EGCG on BMP-4-induced phosphorylation of p70 S6 kinase. However, it was observed that EGCG $(10-30 \mu \mathrm{M})+$ BMP-4 had no significant effect on BMP-4-induced phosphorylation of p70 S6 kinase in comparison to the value of vehicle + BMP-4 (Fig. 7).

\section{Discussion}

In the present study, it was observed that EGCG, which is a primary polyphenolic component of green tea, significantly enhanced BMP-4-stimulated osteoprotegerin release in osteoblast-like MC3T3-E1 cells. By contrast, CGA, a primary polyphenolic component of coffee, had no significant effect on the release of osteoprotegerin. It was also demonstrated that BMP-4-induced expression of osteoprotegerin mRNA was significantly enhanced by EGCG $(10 \mu \mathrm{M})$ in MC3T3-E1 cells. These results suggest that the stimulatory effects of EGCG on BMP-4-induced osteoprotegerin release may be mediated at a transcriptional level. Notably EGCG, but not CGA, may 
upregulate BMP-4-stimulated synthesis of osteoprotegerin in osteoblast-like MC3T3-E1 cells.

It has been established that Smad proteins, as intracellular signaling molecules, typically mediate the effects of the TGF- $\beta$ superfamily, including BMPs (15). In particular, the effects of BMPs are exerted through Smad1/5/8, as a receptor-regulated Smad protein (15). However, the present study observed that EGCG had no significant effect on BMP-4-induced phosphorylation of Smad1 in osteoblast-like MC3T3-E1 cells, indicating that the stimulatory effect of EGCG on BMP-4-induced osteoprotegrin synthesis does not occur through upregulation of the Smad pathway. Recent studies have indicated that Smad-independent pathways, including the MAPK superfamily, may mediate the effects of BMPs in addition to Smad-dependent signaling $(11,12)$. In previous studies by the present authors $(24,26)$, it has been demonstrated that that BMP-4 promotes the activation of p38 MAPK in osteoblast-like MC3T3-E1 cells, resulting in enhanced synthesis of osteocalcin and VEGF. Therefore, the present study evaluated the potential association between p38 MAPK and the effect of EGCG on BMP-4-stimulated osteoprotegerin synthesis. However, EGCG had no significant effect on BMP-induced phosphorylation of p38 MAPK in MC3T3-E1 cells, indicating that the stimulatory effects of EGCG on osteoprotegrin synthesis do not occur through p38 MAPK signaling.

It has also been documented that BMP-4 may induce the activation of p70 S6 kinase in osteoblast-like MC3T3-E1 cells, and that activated p70 S6 kinase may limit BMP-4-stimulated osteocalcin synthesis (30). Based on these findings, the present study evaluated the potential association of p70 S6 kinase with EGCG-enhanced osteoprotegerin synthesis. Whereas EGCG had no significant effect on BMP-4-induced phosphorylation of p70 S6 kinase, it was observed that BMP-4-induced release of osteoprotegerin was suppressed by rapamycin, as an inhibitor of mTOR and established activator of p70 S6 kinase (29). Collectively, these data suggest that EGCG within osteoblasts may act at a point downstream of Smad proteins, p38 MAPK, p70 S6 kinase, and/or other signaling targets, where it is potentially associated with the stimulation of osteoprotegerin synthesis. Future studies are warranted to verify the molecular mechanisms underlying the effects of EGCG on osteoprotegerin synthesis in osteoblasts.

Polyphenolic compounds present in beverages, including green tea and coffee, are considered to have beneficial properties for human health. In the present study, it was demonstrated that EGCG, but not CGA, enhanced BMP-4-stimulated osteoprotegerin synthesis in osteoblast-like MC3T3-E1 cells. EGCG is a catechin that is predominantly present in green tea. Green tea consumption may be associated with increased bone mass, improved bone mineral density and a decreased risk of fracture (13). In addition, BMP-4 is associated with bone formation and serves a key role in the process of fracture healing (16). Conversely, osteoprotegerin serves as a decoy receptor of the RANK ligand and blocks RANK-RANK ligand interactions required for the differentiation and activation of osteoclasts (1). Therefore, the stimulatory effects of EGCG on BMP-4-stimulated osteoprotegerin synthesis may lead to attenuation in bone resorption, resulting in an imbalance in bone metabolism towards increased bone formation. In the present study, EGCG enhanced BMP-4-stimulated osteoprotegerin synthesis at $\geq 3 \mu \mathrm{M}$. In human volunteers, it has been documented that a single EGCG dosage of 1,600 mg/day leads to a maximum plasma concentration of $11.08 \mu \mathrm{M}$ (31). Therefore, the concentration of EGCG used in the present in vitro study may be achievable in vivo.

In conclusion, the present results suggest that EGCG, but not CGA, may enhance BMP-4-stimulated osteoprotegerin synthesis in osteoblasts. The potential modulatory effects of EGCG osteoblast function and bone metabolism may be useful in the prevention of fractures, particularly amongst the elderly. Future studies are now warranted to determine the precise molecular effects of EGCG on bone metabolism.

\section{Acknowledgements}

The authors wish to thank Mrs. Yumiko Kurokawa for her technical assistance. The present study was supported by the Ministry of Education (grant no. 19591042), the Ministry of Health, Labor and Welfare (grant no. H25-Aging-General-004) and the National Center for Geriatrics and Gerontology (grant no. 25-4, 26-12), Japan.

\section{References}

1. Simonet WS, Lacey DL, Dunstan CR, Kelley M, Chang MS, Lüthy R, Nguyen HQ, Wooden S, Bennett L, Boone T, et al: Osteoprotegerin: A novel secreted protein involved in the regulation of bone density. Cell 89: 309-319, 1997.

2. Karsenty $\mathrm{G}$ and Wagner EF: Reaching a genetic and molecular understanding of skeletal development. Dev Cell 2: 389-406, 2002.

3. Zuo C, Huang Y, Bajis R, Sahih M, Li YP, Dai K and Zhang X: Osteoblastgenesis regulation signals in bone remodeling. Osteoporos Int 23: 1653-1663, 2012.

4. Hadjidakis DJ and Androulakis II: Bone remodeling. Ann N Y Acad Sci 1092: 385-396, 2006.

5. Rachner TD, Khosla S and Hofbauer LC: Osteoporosis: Now and the future. Lancet 377: 1276-1287, 2011.

6. Seitz S, Priemel M, Zustin J, Beil FT, Semler J, Minne H, Schinke T and Amling M: Paget's disease of bone: Histologic analysis of 754 patients. J Bone Miner Res 24: 62-69, 2009.

7. Kwan Tat S, Padrines M, Théoleyre S, Heymann D and Fortun Y: IL-6, RANKL, TNF-alpha/IL-1: Interrelations in bone resorption pathophysiology. Cytokine Growth Factor Rev 15: 49-60, 2004.

8. George SE, Ramalakshmi K and Mohan Rao LJ: A perception on health benefits of coffee. Crit Rev Food Sci Nutr 48: 464-486, 2008.

9. Thielecke F and Boschmann M: The potential role of green tea catechins in the prevention of the metabolic syndrome-a review. Phytochemistry 70: 11-24, 2009.

10. Shimizu M, Adachi S, Masuda M, Kozawa O and Moriwaki H: Cancer chemoprevention with green tea catechins by targeting receptor tyrosine kinases. Mol Nutr Food Res 55: 832-843, 2011.

11. Folwarczna J, Pytlik M, Zych M, Cegieła U, Nowinska B, Kaczmarczyk-Sedlak I, Sliwinski L, Trzeciak H and Trzeciak HI: Effects of caffeic and chlorogenic acids on the rat skeletal system. Eur Rev Med Pharmacol Sci 19: 682-693, 2015.

12. Kwak SC, Lee C, Kim JY, Oh HM, So HS, Lee MS, Rho MC and $\mathrm{Oh} \mathrm{J}$ : Chlorogenic acid inhibits osteoclast differentiation and bone resorption by down-regulation of receptor activator of nuclear factor kappa-B ligand-induced nuclear factor of activated T cells c1 expression. Biol Pharm Bull 36: 1779-1786, 2013.

13. Shen CL, Yeh JK, Cao JJ and Wang JS: Green tea and bone metabolism. Nutr Res 29: 437-456, 2009.

14. Singh R, Akhtar N and Haqqi TM: Green tea polyphenol epigallocatechin-3-gallate: Inflammation and arthritis. (corrected). Life Sci 86: 907-918, 2010.

15. Miyazono K, Maeda $\mathrm{S}$ and Imamura T: BMP receptor signaling: Transcriptional targets, regulation of signals, and signaling cross-talk. Cytokine Growth Factor Rev 16: 251-263, 2005. 
16. Krause C, de Gorter DJJ, Karperien M and ten Dijke P: Signal transduction cascades controlling osteoblast differentiation. In: Primer on the Metabolic Bone Diseases and Disorders of Mineral Metabolism. Rosen CJ (eds). 7th edition. John Wiley \& Sons, Inc., Washington, DC, 2008.

17. Moustakas A and Heldin CH: Non-Smad TGF-beta signals. J Cell Sci 118: 3573-3584, 2005.

18. Cai J, Pardali E, Sánchez-Duffhues G and ten Dijke P: BMP signaling in vascular disease. FEBS Lett 586: 1993-2002, 2012.

19. KuroyanagiG, TokudaH, YamamotoN,Matsushima-NishiwakiR Mizutani J, Kozawa O and Otsuka T: Resveratrol amplifies BMP-4-stimulated osteoprotegerin synthesis via p38 MAPK in osteoblasts. Mol Med Rep 12: 3849-3854, 2015.

20. Sudo H, Kodama HA, Amagai Y, Yamamoto S and Kasai S: In vivo differentiation and calcification in a new clonal osteogenic cell line derived from newborn mouse calvaria. J Cell Biol 96: 191-198, 1983.

21. Kozawa O, Tokuda H, Miwa M, Kotoyori J and Oiso Y: Cross-talk regulation between cyclic AMP production and phosphoinositide hydrolysis induced by prostaglandin E2 in osteoblast-like cells Exp Cell Res 198: 130-134, 1992.

22. Simpson DA, Feeney S, Boyle C and Stitt AW: Retinal VEGF mRNA measured by SYBR green I fluorescence: A versatile approach to quantitative PCR. Mol Vis 6: 178-183, 2000.

23. Pryor RJ and Wittwer CT: Real-time polymerase chain reaction and melting curve analysis. Methods Mol Biol 336: 19-32, 2006.

24. Kozawa O, Hatakeyama D and Uematsu T: Divergent regulation by $\mathrm{p} 44 / \mathrm{p} 42$ MAPK and p38 MAPK of bone morphogenetic protein-4-stimulated osteocalcin synthesis in osteoblasts. J Cell Biochem 84: 583-589, 2002.
25. Kozawa O, Matsuno $\mathrm{H}$ and Uematsu T: Involvement of p70 S6 kinase in bone morphogenetic protein signaling: Vascular endothelial growth factor synthesis by bone morphogenetic protein- 4 in osteoblasts. J Cell Biochem 81: 430-436, 2001.

26. Tokuda H, Hatakeyama D, Shibata T, Akamatsu S, Oiso Y and Kozawa O: P38 MAPK regulates BMP-4-stimulated VEGF synthesis via p70 S6 kinase in osteoblasts. Am J Physiol Endocrinol Metab 284: E1202-E1209, 2003.

27. Laemmli UK: Cleavage of structural proteins during the assembly of the head of bacteriophage T4. Nature 227: 680-685, 1970.

28. Kato K, Ito H, Hasegawa $\mathrm{K}$, Inaguma $\mathrm{Y}$, Kozawa $\mathrm{O}$ and Asano T: Modulation of the stress-induced synthesis of hsp27 and alpha B-crystallin by cyclic AMP in C6 glioma cells. J Neurochem 66: 946-950, 1996.

29. Li Y, Corradetti MN, Inoki K and Guan KL: TSC2: Filling the GAP in the mTOR signaling pathway. Trend Biochem Sci 29: 32-38, 2004.

30. Minamitani C, Tokuda H, Adachi S, Matsushima-Nishiwaki R, Yamauchi J, Kato K, Natsume H, Mizutani J, Kozawa O and Otsuka T: P70 S6 kinase limits tumor necrosis factor-alpha-induced interleukin-6 synthesis in osteoblast-like cells. Mol Cell Endocrinol 315: 195-200, 2010.

31. Ullmann U, Haller J, Decourt JP, Girault N, Girault J, Richard-Caudron AS, Pinceau B and Weber P: A single ascending dose study of epigalocatechin gallate in healthy volunteers. J Int Med Res 31: 88-101, 2003. 